\title{
Visuomotor associations facilitate movement preparation
}

David M. Huberdeau \& Nicholas B. Turk-Browne

Department of Psychology, Yale University

Main text: 5417 words

Please address correspondence to:

David Huberdeau

Department of Psychology

Yale University

2 Hillhouse Ave

New Haven, CT 06511

david.huberdeau@yale.edu 


\begin{abstract}
Motor behavior can benefit from discovering and remembering perceptual cues that reliably predict the appropriateness and requirements of a movement. Here we investigate the use and learning of these visuomotor associations. We use a powerful experimental technique that controls response time, allowing us to investigate the effects of perceptual cues on movement planning without a speed-accuracy trade-off. In Experiment 1, participants first memorized associations between symbols and movement goals. When these symbols were subsequently presented as cues, reaching movements were prepared as efficiently as if the goals themselves were previewed, without the need for additional practice. In Experiment 2, participants had to learn these visuomotor associations during the movement task itself. This learning enabled efficient movement preparation, and the rate of improvement scaled with the number and complexity of associations. These findings suggest that movement preparation can be facilitated by perceptual cues via statistical learning and memory recall, highlighting a potential role for learning and memory systems not conventionally implicated in motor behavior.
\end{abstract}

\title{
Keywords
}

statistical learning, reaching behavior, motor control, action and perception, memory 


\section{Public significance statement}

What do we learn when we learn a new skill? The most obvious answer is that we learn how to generate movements that were previously unfamiliar to us. A less obvious but critical answer, investigated in this study, is that we learn the perceptual events that occur before and during the execution of a skill, and then use those events to better prepare for upcoming movements. 


\section{Introduction}

Consider the problem faced by baseball batters: they have very little time to plan their swing given the speed and distance of pitches, and so must rely on subtle perceptual cues to guide motor preparation, such as the angle of the pitcher's arm, the hand grip, and the position and spin of the ball (Bahill et al., 2005). In the laboratory, this kind of learning behavior can be studied with visuomotor association (VMA) tasks, in which human participants must remember or learn how to respond to the appearance of an arbitrary perceptual cue. There are a number of variants of the VMA task but typically, a visual cue (e.g., a letter or a shape) is presented followed by a forced-choice task in which participants select one among several targets (Asaad et al., 1998; Murray \& Wise, 1996). The visual cue predicts the subsequent appearance of a particular movement target and participants learn these associations through experience. Learning in these tasks is usually inferred by observing participant's choice accuracy and response time (RT).

The VMA task provides the opportunity to study the bidirectional interaction of cognition and movement. This has revealed how cognitive processes can influence motor preparation, including highlighting a role for different memory systems (Knowlton et al., 1996). Likewise, examining choice behavior and movement in the same task allows for an assessment of motor factors in cognitive performance. For example, even a response as simple as a two-choice button press can be contaminated by idiosyncrasies of movement, such as a difference in response latency when switching from one hand to the other across trials (Trapp et al., 2012). Carefully controlling and measuring the kinematics of movement as the output behavior after a choice allows researchers to better understand complex behavior. 
Despite these strengths, speed-accuracy tradeoffs can occur in the VMA task, introducing potential confounds in interpreting performance (Liu \& Watanabe, 2012; Pew, 1969; Wood \& Jennings, 1976). For instance, different memory mechanisms or cognitive strategies might be responsible for choice behavior when the RT is short compared to long (Dang et al., 2018; McElree et al., 2006; Peebles \& Bothell, 2014). Furthermore, the mechanism underlying behavior might change with practice such that higher choice accuracies are possible at shorter RTs (Hardwick et al., 2017). This type of transformation could be obscured if RTs are not carefully controlled, as participants may maintain a longer RT than necessary (Haith et al., 2016), which can happen out of habit (Wong et al., 2017).

In addition to speed-accuracy tradeoffs, it can also be unclear how learning enhances performance in the VMA task. Learning to associate an action with a visual cue is likely a multicomponent process (Bédard \& Sanes, 2009; Collins \& Frank, 2012; Huberdeau, Krakauer, et al., 2015; Knowlton et al., 1996; McDougle \& Taylor, 2019; Squire \& Zola, 1996). Participants might rely on explicit memory for the associations (Squire, 1992) or more implicit mechanisms such as reinforcement learning (Doll et al., 2015; Sutton \& Barto, 1998) or statistical learning (Hunt \& Aslin, 2001; Nissen \& Bullemer, 1987).

Here, we sought to take advantage of the benefits of the VMA task while controlling for potential confounds in order to determine the learning and memory mechanisms responsible for movement preparation. We report two experiments that carefully control RT while quantifying the kinematics of movement during a VMA task. This design allowed us to relate the extent of learning to the extent of movement preparation across RTs. In Experiment 1, arbitrary associations between visual cues and movement goal locations were memorized in advance of 
the movement task. In Experiment 2, these associations had to be learned through experience during the movement task itself. In both experiments, we employed a powerful behavioral method that made it possible to approximately control response latencies, effectively making RT an independent variable (Ghez et al., 1997; Haith et al., 2015, 2016). This method allowed us to measure response accuracy and movement kinematics as a function of the amount of time available to prepare the response (preparation time, PT). Thus, we avoided the aforementioned challenges inherent in using either RT or choice accuracy alone as a dependent measure.

Combined with this approach, we introduce a variant of the VMA task in which the movement onset is delayed relative to an informative pre-cue to investigate the consequences of memory recall on movement preparation, and to study the nature of learning of visuomotor associations. We hypothesized that when symbol-target associations were memorized ahead of time, participants would use knowledge of the associations to prepare movements to a future target just as efficiently as when the target was previewed by directly cuing its location. Furthermore, we hypothesized that learning the associations through cross-situational regularities (Aslin \& Newport, 2012; Frank et al., 2007; Yu \& Smith, 2007) would gradually reveal facilitation of movements by symbols.

\section{Methods}

\section{Participants}

A total of 95 undergraduate students were recruited for this study. The study was approved by the Yale Institutional Review Board and all participants provided informed consent prior to participation. 


\section{Apparatus}

Participants made right upper-limb planar reaching movements while seated at a table. A computer monitor (Dell P2717H) positioned vertically approximately $90-100 \mathrm{~cm}$ in front of the participant displayed in real-time a cursor (black dot $0.64 \mathrm{~cm}$ diameter) representing the position of their hand. A webcam (4 Mega Pixel, 60 frames per second, Kayeton Technology Co., Shenzhen, China) positioned $53 \mathrm{~cm}$ above the surface of the table recorded the kinematics of movement at $60-\mathrm{Hz}$ by detecting the location of a colored marking warn on the participant's hand (Figure 1a).

\section{General procedure}

Reaches were planar, began at a central location on the table, and were directed towards targets represented as filled gray circles $(1.25 \mathrm{~cm}$ diameter) on the display. Movements on the table needed to exceed $11-\mathrm{cm}$ from the start position for the cursor to reach or exceed the target on the display. Participants were instructed to make "shooting" movements and not to stop their reach on the target. Possible target locations differed by experiment as described in detail below. A trial began when the cursor was positioned within $2.5 \mathrm{~mm}$ of the start position. Participants were instructed and trained to initiate their movement coincident with the third auditory tone in a sequence of three tones, "the metronome" (first two tones: $1 \mathrm{kHz}$ monotone of $0.1 \mathrm{~s}$ duration; third tone: $1.7 \mathrm{kHz}$ monotone of $0.1 \mathrm{~s}$ duration; $0.4 \mathrm{~s}$ inter-tone-interval). The target for each trial was presented a variable amount of time $(0.25-0.80 \mathrm{~s})$ prior to the designated movement initiation time (Figure 1b). Varying the target presentation time parameterized the amount of PT available to view the location of the movement goal prior to movement initiation. Participants

were trained to prioritize having accurate movement initiation times, even when the 
presentation of the target occurred only briefly before the third tone. Participants were

Figure 1

a

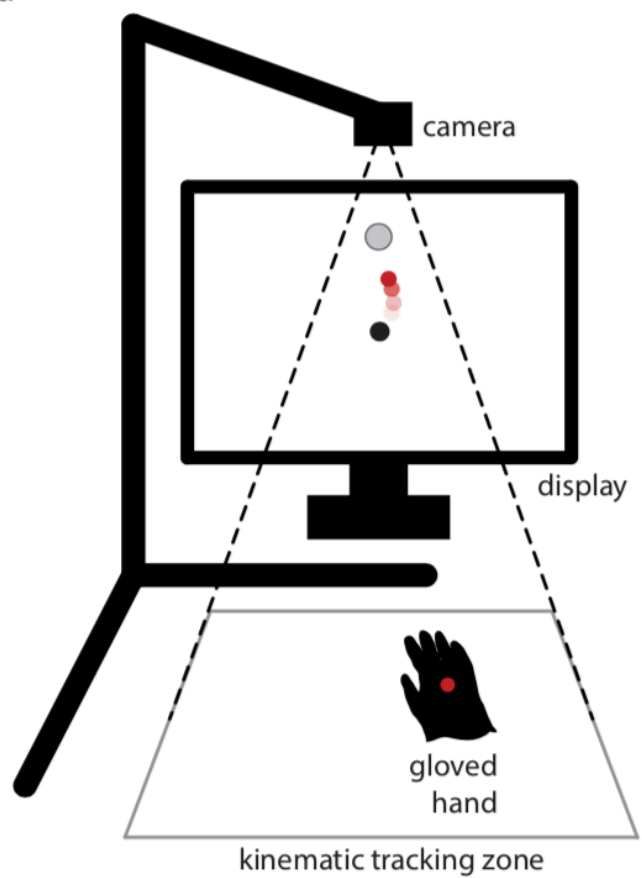

b
C

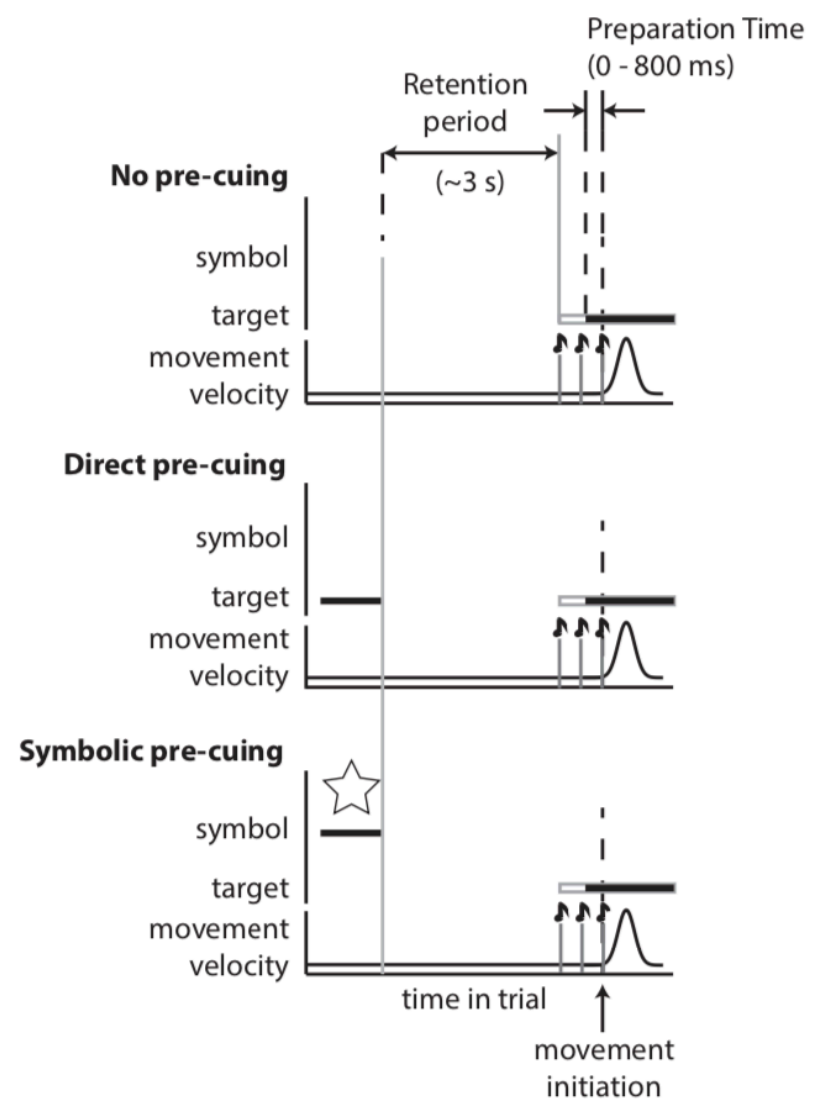

Figure 1: Schematic of apparatus and task. (a) The kinematics of planar reaching movements were measured using a digital camera that detected the location of a colored marking on an individual's hand and displayed that location to the participant in real time (red cursor on the computer screen). (b) The mapping showing which symbol was associated with each target for Experiment 1. The symbols are displayed at the target location only for visualization purposes, they appeared at the center of the screen over the start position during the experiment. (c) Trials consisted of either No pre-cuing, Direct pre-cuing, or Symbolic pre-cuing. Horizonal bars indicate times in the trial during which the symbol or the target were displayed. Musical notes indicate times in the trial during which auditory tones were presented. 
instructed to choose a reach direction even if they were unable to detect the target location by the time of the third auditory tone.

The experiment contained three trial conditions (Figure 1b). For No pre-cue trials, a wait period of $3.25 \mathrm{~s}$ was imposed prior to the start of the metronome. For Direct pre-cue trials, the target was displayed for $0.25 \mathrm{~s}$, followed by a wait period of $3 \mathrm{~s}$. For Symbolic pre-cue trials, a symbol (Figure 1c) was displayed at the center of the screen covering the start position for 0.25 s followed by a 3-s wait period. For both Direct and Symbolic trials, the target that ultimately appeared was consistent with the pre-cue on $90 \%$ of trials; when a different target appeared than was initially indicated (a catch trial), it was selected with equal probability among the remaining possible target locations. Trials were divided into blocks, with each block lasting 8.7 minutes on average. Catch trials did not occur during the first 10 trials of any block.

\section{Experiment 1}

In the first experiment, 21 participants memorized associations between symbols and target locations before the experiment began. A mapping of the associations (Figure 1c) was shown to the participants on a sheet of paper and they were given as much time as needed to view this mapping (typically less than $1 \mathrm{~min}$ ) before beginning a task practice session of 12 No pre-cue trials. Following the practice session, participants were presented with a test of memory for the associations that consisted of identifying the symbol associated with each target from a list of symbols that included never-before-seen symbols. Following the practice block and assessment of memory for the symbol-target associations, there were three main blocks of 60 trials each. For these blocks, the three trial types were pseudo-randomly interleaved such that there was an average of 20 trials of each type, including catch trials. 


\section{Experiment 2}

In the second experiment, participants were not shown the symbol-target associations prior to the start of the task. They had to acquire these associations online via cross-situational learning (Aslin \& Newport, 2012; Frank et al., 2007; Smith \& Yu, 2013; Yu \& Smith, 2011), where each target was associated with more than one symbol across trials. The first block served as a practice session with 60 trials of the No pre-cue condition. The next four main blocks contained 60 trials, with 27 trials each of Direct and Symbolic trial types, as well as three catch trials of each type. A post-test memory assessment for the symbol-target associations was given at the conclusion of the experiment. There were three different versions of Experiment 2 that sought to manipulate the complexity of learning across participants (all naïve): three targets with two symbols each ( $\mathrm{N}$ $=23)$, four targets with three symbols each $(N=20)$, and six targets with two symbols each $(N=$ 14). Symbols differed across these variants of the experiment and differed in visual complexity from simple geometric shapes to arbitrary symbols and amorphous shapes (Figure 4). A near replication of the three-target variant of Experiment $2(N=17)$ was performed after the data above had been collected and analyzed, and additionally included a memory test for the associations following the first block of symbol-target association learning.

The sample size for Experiment 1 was chosen based on expected effect sizes from similar past work, e.g. (Haith et al., 2015). An estimate of power given the observed effect size from Experiment 1 was computed as above 0.99 , and it was determined that a sample size of 8 would be sufficient for a power of 0.8 .

Data analysis and statistics 
Data were analyzed using Matlab (The Mathworks, Natick, MA) and R (The R Project, https://www.R-project.org/). The PT for each trial was computed as the difference between when the target appeared and the time at which the distance between the start position and the cursor first exceeded $0.5 \mathrm{~cm}$. The reach direction for each trial was computed by measuring the angle of the movement velocity when the reach was $3 \mathrm{~cm}$ from the start position. Reaches were labelled as correct when the angular reach was within $30^{\circ}$ of the target direction.

These kinematic data were then used to assess the probability of choosing the correct target (the "success probability"), the peak velocity, and the variability of reach-directions toward the chosen target (the "directional variability"), as functions of PT. These measures were used to ascertain the state of movement preparation under the three trial types and in catch trials across preparation times. Linear mixed-effects models were fit to each of these dependent variables as functions of PT and trial type, and a likelihood ratio test was conducted to test for main effects and interactions. Post-hoc analyses were conducted to test whether success probability, peak velocity, or directional variability varied by PT for each trial type.

An individual's minimum PT was computed according to the formula below (see also Supplemental Figure 1):

$$
\min P T=\underset{t}{\operatorname{argmax}}(\operatorname{Pr}(\text { successful|PT }>t)+\operatorname{Pr}(\text { unsuccessful|PT<t }))
$$

This automated determination of minimum PT was manually verified and participants were excluded if the determination appeared incorrect, usually on account of insufficient sampling across PTs because of participant non-compliance with instructions. One participant (5\%) was excluded for this reason from Experiment 1 and 10 total (14\%) from all conditions in Experiment 2 and the replication. 
The key analyses and measures were mostly the same for Experiments 1 and 2, primarily reach direction and success on each trial. The probability of making a correct reach among reaches with a PT less than the individual's minimum PT was computed for each half-block, which comprised 30 trials. Reaches with PTs greater than an individual's minimum PT were excluded from this analysis to isolate trials that must necessarily have depended on learned associations. That is, reaches with sufficient PT could have been successful even if the association between the symbols and targets was unknown because participants could have responded to the appearance of the target rather than pre-emptively preparing a movement to the target in response to the pre-cue. Thus, excluding trials with high-PT provides a more accurate estimate of the ability of participants to use associations for movement preparation across the experiment.

Experiment 2 additionally permitted us to ascertain the process of learning the symboltarget associations. In this analysis, the probability of reach success when a reach was conducted with a PT less than an individual's minimum PT was computed as a function of the number of times that an individual cue occurred in the experiment. This analysis was performed separately for Symbolic and Direct pre-cue trials and was repeated using data from Experiment 1 (where learning across symbol-appearances was not expected) as a comparison and baseline for learning in Experiment 2. Exponential functions of the form:

$$
y=\left(1-\beta e^{-\alpha t}\right)
$$

were fit to success probability data, where $y$ was taken to be the success probability, $t$ the number of occurrences of a symbol, $\beta$ the offset of the intercept (i.e. the success probability on the first trial), and $\alpha$ the rate of learning. 
Catch trials (where a different target appeared than had been cued) were analyzed in the same way as all other trials. For both Experiments 1 and 2, an analysis was conducted to determine whether additional PT was required to initiate movements to the presented target during catch trials. To test for this, a general linear model that used a logistic link function was fit to success-probability data as a function of PT, and a Wald test was used to test whether the parameters for PT, trial type, or their interaction were significant in the model. This analysis was conducted across all trials of Experiments 1 and 2, with an additional factor for trial number to account for potential learning effects.

Across both Experiments 1 and 2, five independent groups of participants prepared reaches to targets with varying numbers of potential alternatives. This design allowed us to test for any potential relationship between the number of choice alternatives and the minimum time required to prepare a successful movement (the minimum preparation time). A combined analysis was conducted using data pooled from each experiment to test whether there was a relationship between an individual's minimum PT and the number of targets.

\section{Results}

\section{Experiment 1}

We hypothesized that movements would be prepared in advance of their onset when arbitrary cues were presented. Furthermore, we predicted that the pre-cue should enable movement preparation to the impending target regardless of the latency between the target's appearance and the onset of movement. When no pre-cue was presented, however, we expected that 
additional PT would be needed for reaches to match the characteristics of movements that had been pre-cued.

Reaches (Figure 2a) differed in their success probability across PT and by trial types (Figure $2 b)$. There was a significant main effect of PT $\left(X^{2}(1)=79, p<0.001\right)$, a significant main effect of trial type $\left(X^{2}(2)=164, p<0.001\right)$, and a significant interaction between $\mathrm{PT}$ and trial type $\left(\mathrm{X}^{2}(2)=\right.$ 130, $p<0.001)$. A linear mixed effects model fit to data from trials with no pre-cue revealed that the success probability had a large and significant dependence on PT (effect size: $1.3, X^{2}(1)=92$,

Figure 2

a

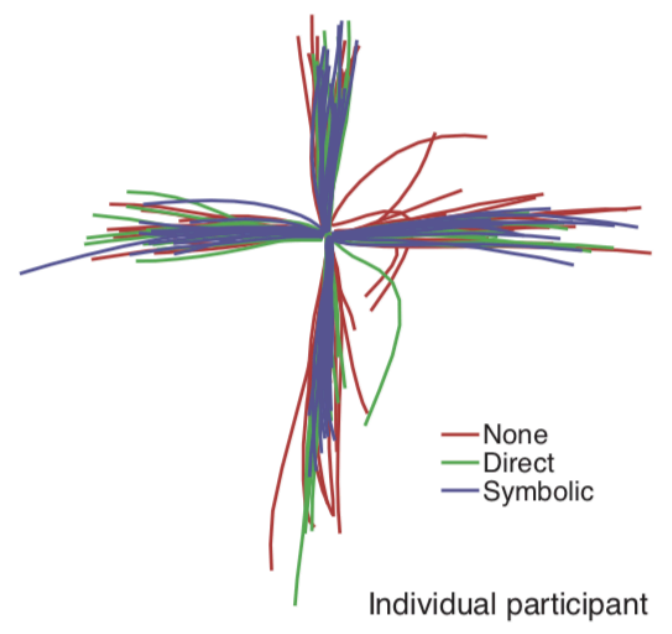

$10 \mathrm{~cm}$ b

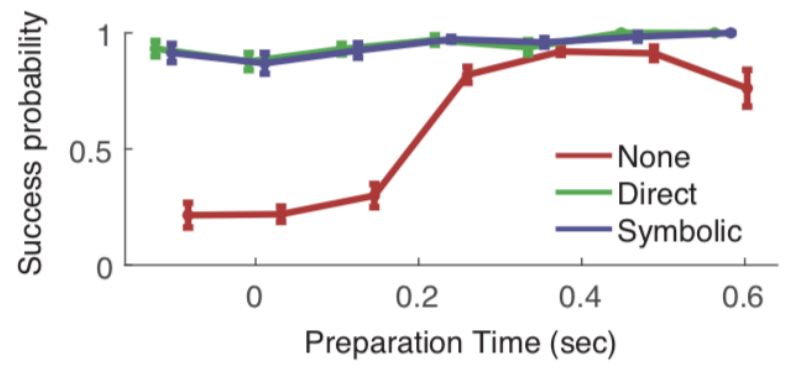

C
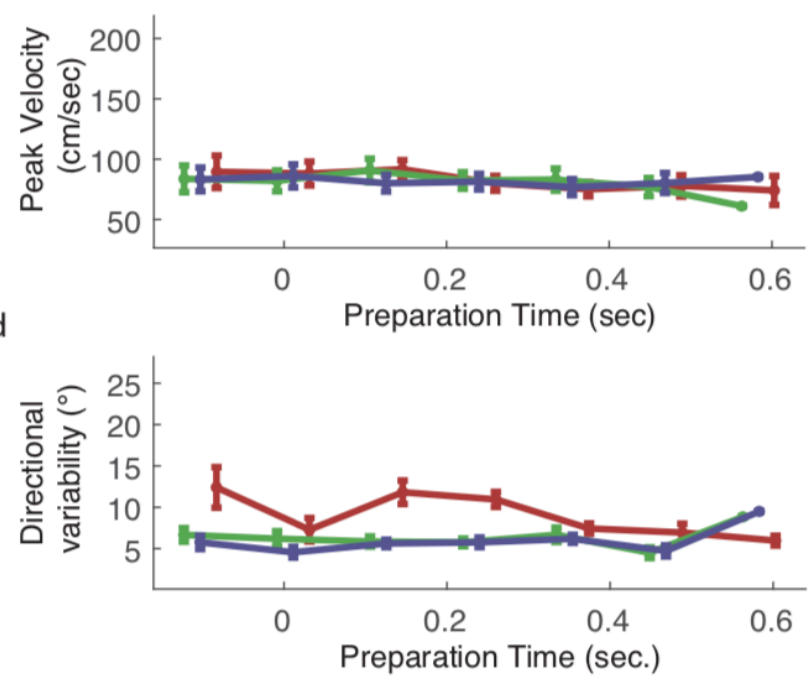

Figure 2: Symbolic and Direct pre-cuing both facilitate efficient movement preparation in Experiment 1. (a) Reaches of one participant showing typical kinematics. The group average for (b) success probability, (c) peak velocity, and (d) directional variability, as a function of preparation time. 
$p<0.001)$. In contrast, direct pre-cued trials and symbolic pre-cued trials had much smaller effect sizes relative to the no pre-cue condition, although the effects of PT were significant (effect size, direct: $0.11, X^{2}(1)=3.9, p=0.047$; effect size, symbolic: $\left.0.10, X^{2}(1)=4.3, p=0.038\right)$. These analyses confirm that the success probability differed by trial type, where reaches with a symbolic or direct pre-cue were similarly successful at preparing the correct reach with only a small effect of PT, while trials without a pre-cue had a large and significant dependence on PT.

Despite the effects of trial type and preparation time on success probability, reaches were kinematically similar across preparation times and trial types (Figure $2 \mathrm{c}$ ). There were no significant main effects on peak velocity $\left(P T: X^{2}(1)=1.5, p=0.23\right.$; trial type: $\left.X^{2}(2)=1.2, p=0.56\right)$, nor an interaction between PT and trial type $\left(\mathrm{X}^{2}(2)=1.1, \mathrm{p}=0.57\right)$.

Directional variability differed across trial types in a similar way to success probability (Figure $2 \mathrm{~d})$. There were significant main effects of PT $\left(X^{2}(1)=4.1, p=0.044\right)$ and trial type $\left(X^{2}(2)\right.$ $=76, p<0.001)$, and a significant interaction between PT and trial type $\left(X^{2}(2)=12, p<0.01\right)$. Directional variability for no pre-cue trials significantly depended on PT (effect size: $-6.2, X^{2}(1)=$ 8.0, $p<0.01$ ), while the directional variability of directly cued trials (effect size: $-0.28, X^{2}(1)=$ $0.054, p=0.82$ ) and symbolically cued trials (effect size: $1.3, X^{2}(1)=1.4, p=0.23$ ) did not. These results are consistent with our hypothesis that direct pre-cues and memorized arbitrary symbolic pre-cues are equally effective at facilitating movement preparation irrespective of PT. In this case, the state of movement preparation is reflected in the variability in initial reach direction.

Did switching the location of the target relative to the cue on catch trials impede movement to the ultimate appearance of the target? An analysis of success probability revealed that catch trials (Figure 3b) depended on PT differently than the other trial types (Figure 3c). A 
Figure 3

a

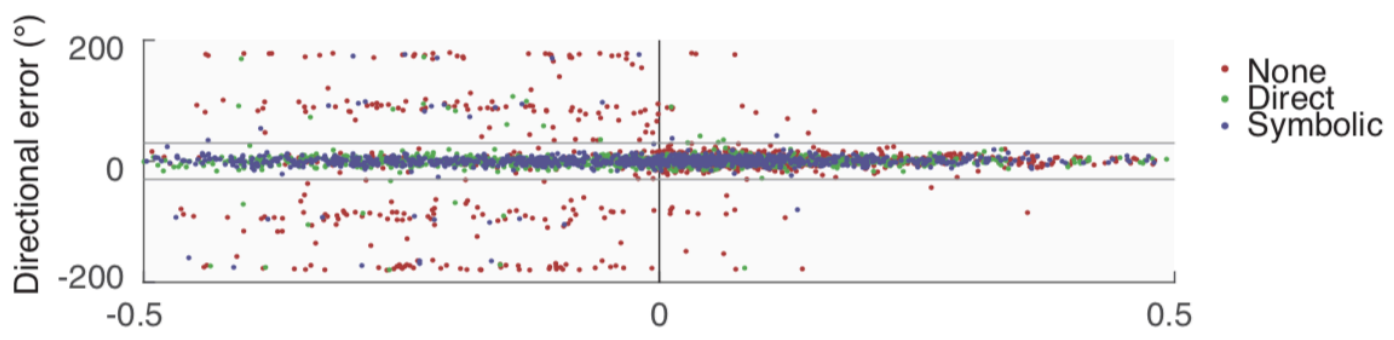

b

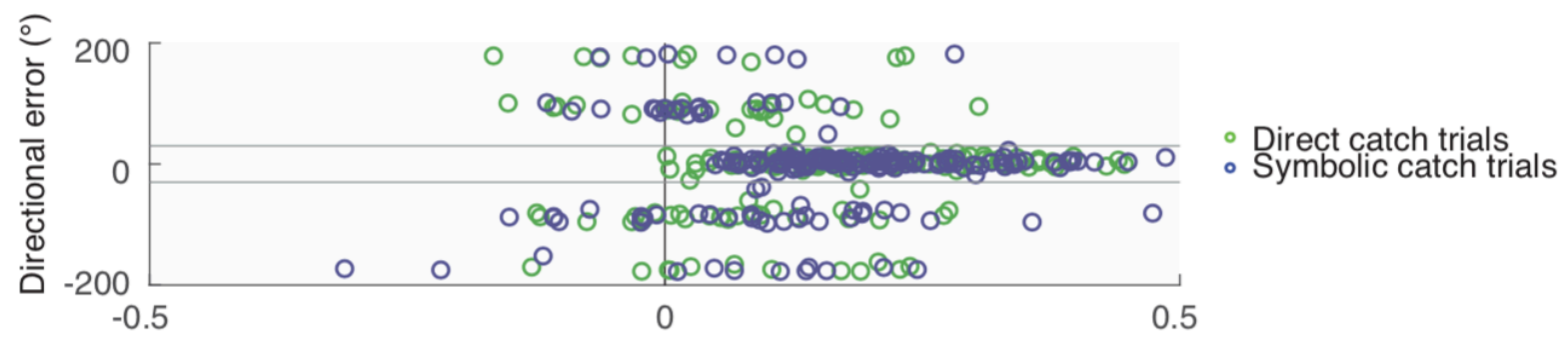

C

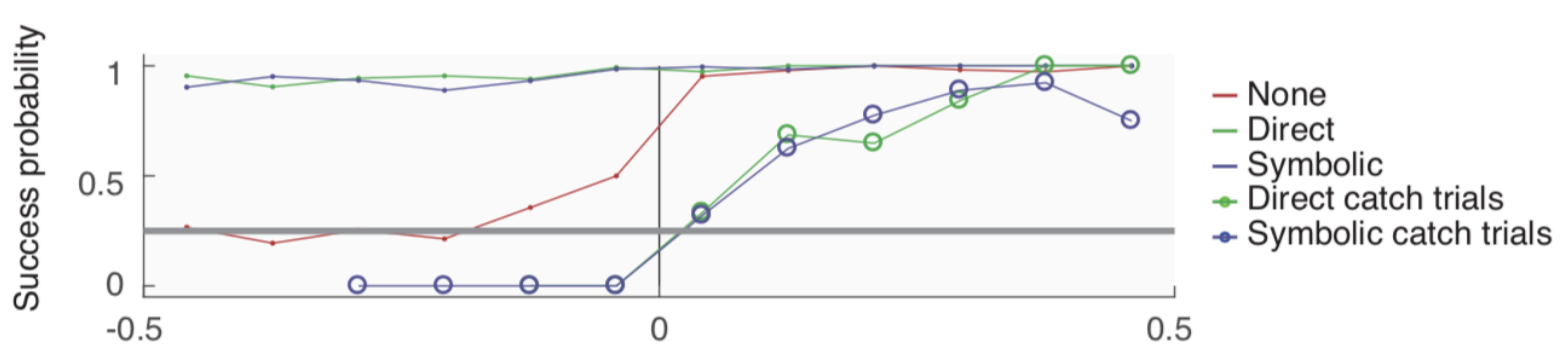

d

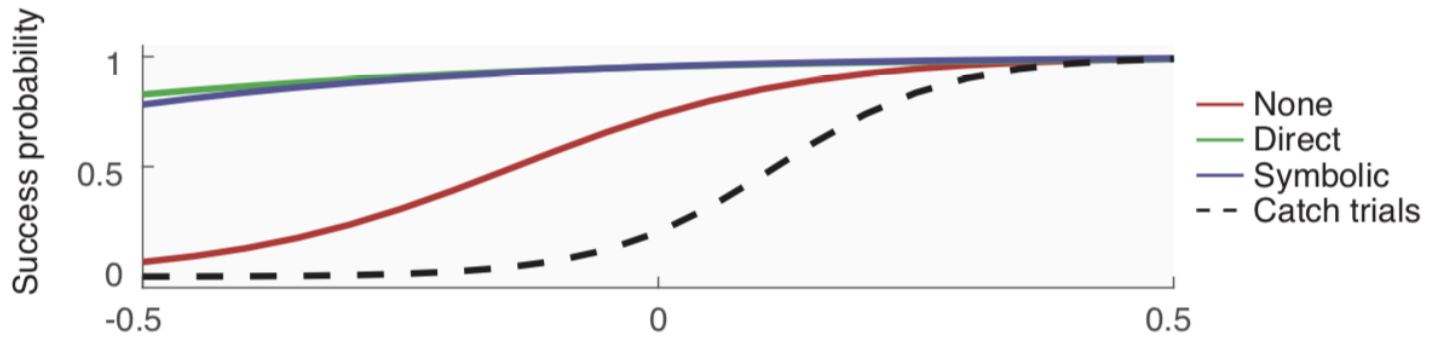

Time from individual minimum preparation time (sec.)

Figure 3: Preparation time data aligned to each individual's minimum PT in Experiment 1. (a) Reach direction as a function of time from the minimum PT and trial type. (b) Same for catch trials. (c) The probability of making a correct reach as a function of the time from the minimum PT and trial type plus catch trials. (d) Sigmoidal fits to each trial type and to catch trials.

general linear model with a logistic link function was fit to binary data (i.e. the reach success of each trial) as a function of PT and trial type (Figure 3d). This model revealed a significant effect of PT (effect size: 7.3, $z=15, p<0.001$ ) and a significant interaction between PT and trial type (Wald test: $\left.X^{2}(3)=57.1, p<0.001\right)$. Critically, participants were less likely to successfully reach 
toward the target on catch trials compared to no cue trials (effect size: $-2.4, z=3.2, p<0.001$ ). This analysis confirmed that catch trials significantly delayed the time at which reaches were successful.

\section{Experiment 2}

Rather than explicitly memorizing visuomotor associations, the participants in this experiment had to extract them in a cross-situational learning design. Across trials, each symbol was reliably paired with a particular target location. We expected that these pairings would need to be learned gradually because more than one symbol could be associated with each target location and there were multiple target locations with which symbols could be associated. To test the speed and capacity of learning visuomotor associations, we varied the number of symbols per target location and the total number of target locations. We tested the hypothesis that statistical learning can form visuomotor associations that facilitate movement preparation, at least for the simpler variants of this experiment involving fewer symbols and target locations.

When two symbols were paired with each of three target locations (Figure 4a), reaches (Figure 4b) with PT lower than an individual's minimum PT had a success probability that increased across blocks (Figure 5a). Linear mixed effects models of success probability across blocks of trials revealed significant main effects of block $\left(X^{2}(1)=8.5, p<0.01\right)$ and trial type $\left(X^{2}(1)\right.$ $=62, p<0.001)$, and a significant interaction between block and trial type $\left(X^{2}(1)=16, p<0.0001\right)$. This demonstrates that learning occurred across blocks, but that the progression was different for Direct and Symbolic trial types. The replication of this experiment (Figure 5b) revealed similar results: there was a significant main effect of trial type $\left(X^{2}(1)=45, p<0.001\right)$ and a significant interaction between block and trial type $\left(X^{2}(1)=11, p<0.001\right)$. The main effect of block did not 
Figure 4

a
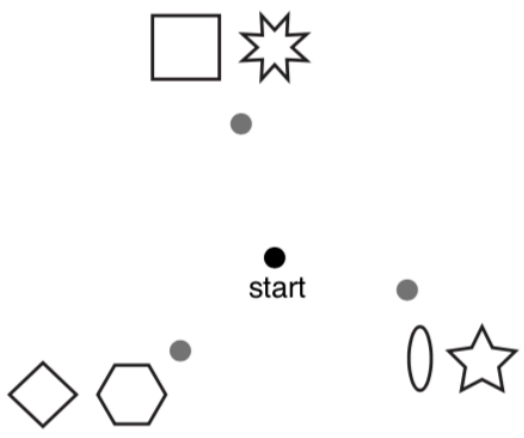

b

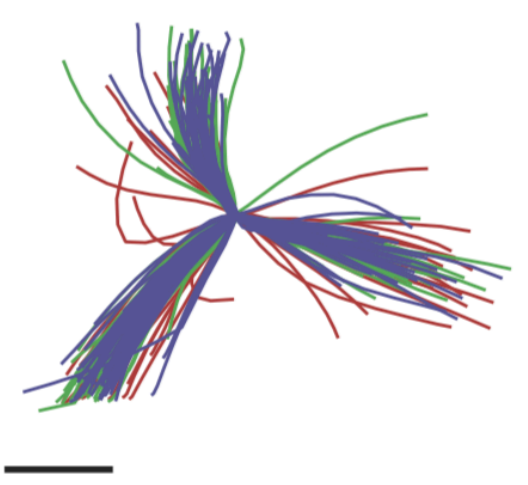

$10 \mathrm{~cm}$

c

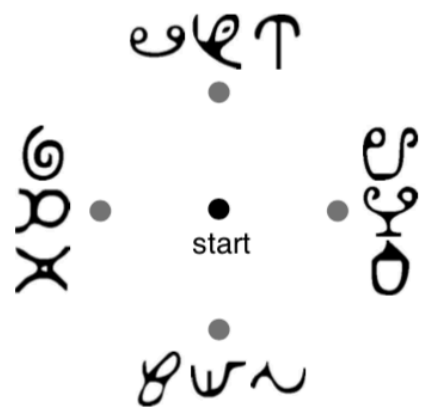

d

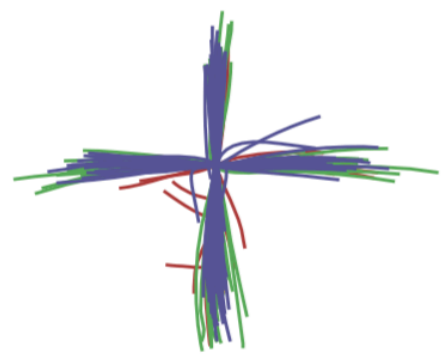

Individual participants e

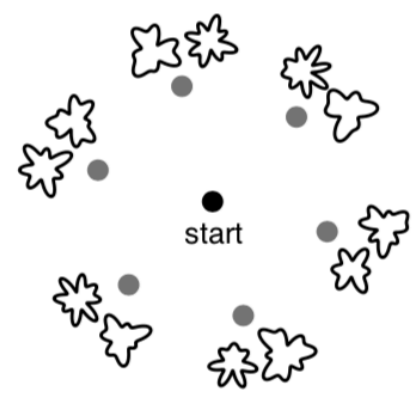

f

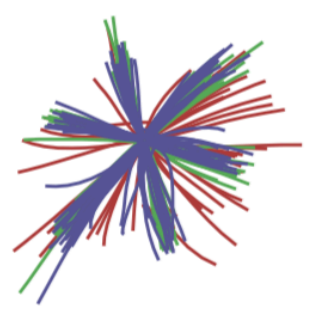

Figure 4: Target locations (gray circles) and symbol mappings (presented in periphery for visualization, always displayed at center) for the variants of Experiment 2 with (a) three targets and six symbols, (c) four targets and twelve symbols, and (e) six targets and twelve symbols. $(b, d, f)$ Reaches for one participant in each variant showing example kinematics.

reach significance $\left(X^{2}(1)=2.7, p=0.10\right)$. However, as suggested by the interaction, analyses of each trial type revealed a significant main effect of block for Symbolic trials $\left(X^{2}(1)=9.4, p<0.01\right)$, but not Direct trials $\left(X^{2}(1)=2.1, p=0.14\right)$. Thus, in both the original study and the replication study, there was evidence of learning across trials for the Symbolic pre-cue condition.

The variant with four targets and three symbols per target (Figures $4 c-d$ ) exhibited the same pattern of results as above (Figure $5 c)$. There were significant main effects of block $\left(X^{2}(1)=\right.$ $16, p<0.001)$ and trial type $\left(X^{2}(1)=150, p<0.001\right)$, and a significant interaction between block and trial type $\left(X^{2}(1)=13, p<0.001\right)$, demonstrating a learning effect across practice for the 
Figure 5
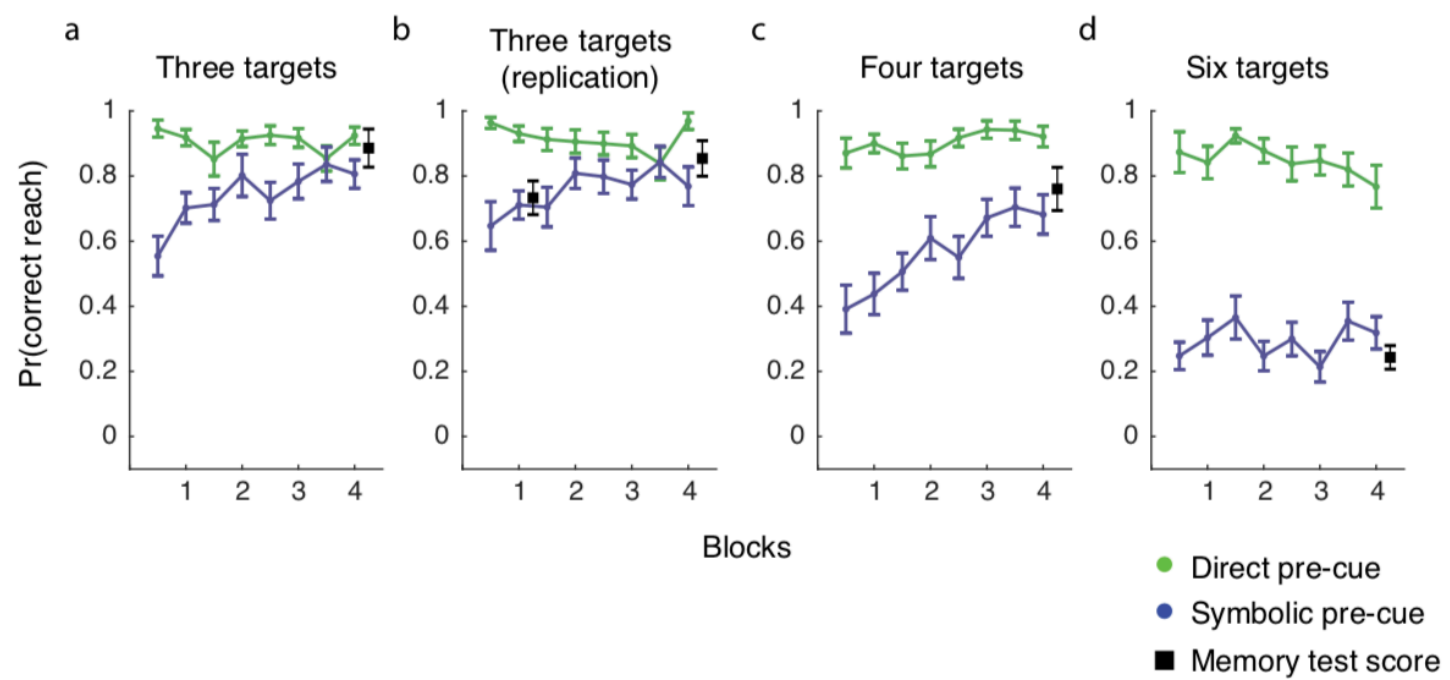

Figure 5: Probability of making a correct reach among movements with a PT below the minimum PT in Experiment 2. Black squares indicate the group average memory test score. Results for (a) the three-target variant, (b) the three-target replication, (c) the four-target variant, and (d) the six-target variant.

Symbolic condition. In the variant with six targets and two symbols per target (Figures 4e-f), however, there was no evidence of learning (Figure $5 \mathrm{~d}$ ): despite a significant main effect of trial type $\left(X^{2}(1)=260, p<0.0001\right)$, there was no main effect of block $\left(X^{2}(1)=0.27, p=0.60\right)$, nor of the interaction between block and trial type $\left(X^{2}(1)=2.3, p=0.13\right)$.

The success probability, given that the reach had a PT below the participant's minimum PT, was measured as a function of the total number of times that a symbol was encountered by the participant (Figure 6). Exponential functions fit to success probability revealed significant effects in the three-target variant $(\beta=0.81, \mathrm{t}=41, \mathrm{p}<0.0001 ; \alpha=-0.59, \mathrm{t}=6.4, \mathrm{p}<0.0001)$ and its replication $(\beta=0.79, \mathrm{t}=41, \mathrm{p}<0.0001 ; \alpha=-1.3, \mathrm{t}=3.3, \mathrm{p}<0.01)$, where $\beta$ represents the initial success probability and $\alpha$ represents the rate of learning, with larger absolute magnitudes reflecting faster learning. Similar effects were found for the four-target variant $(\beta=0.60, t=17$, 
Figure 6
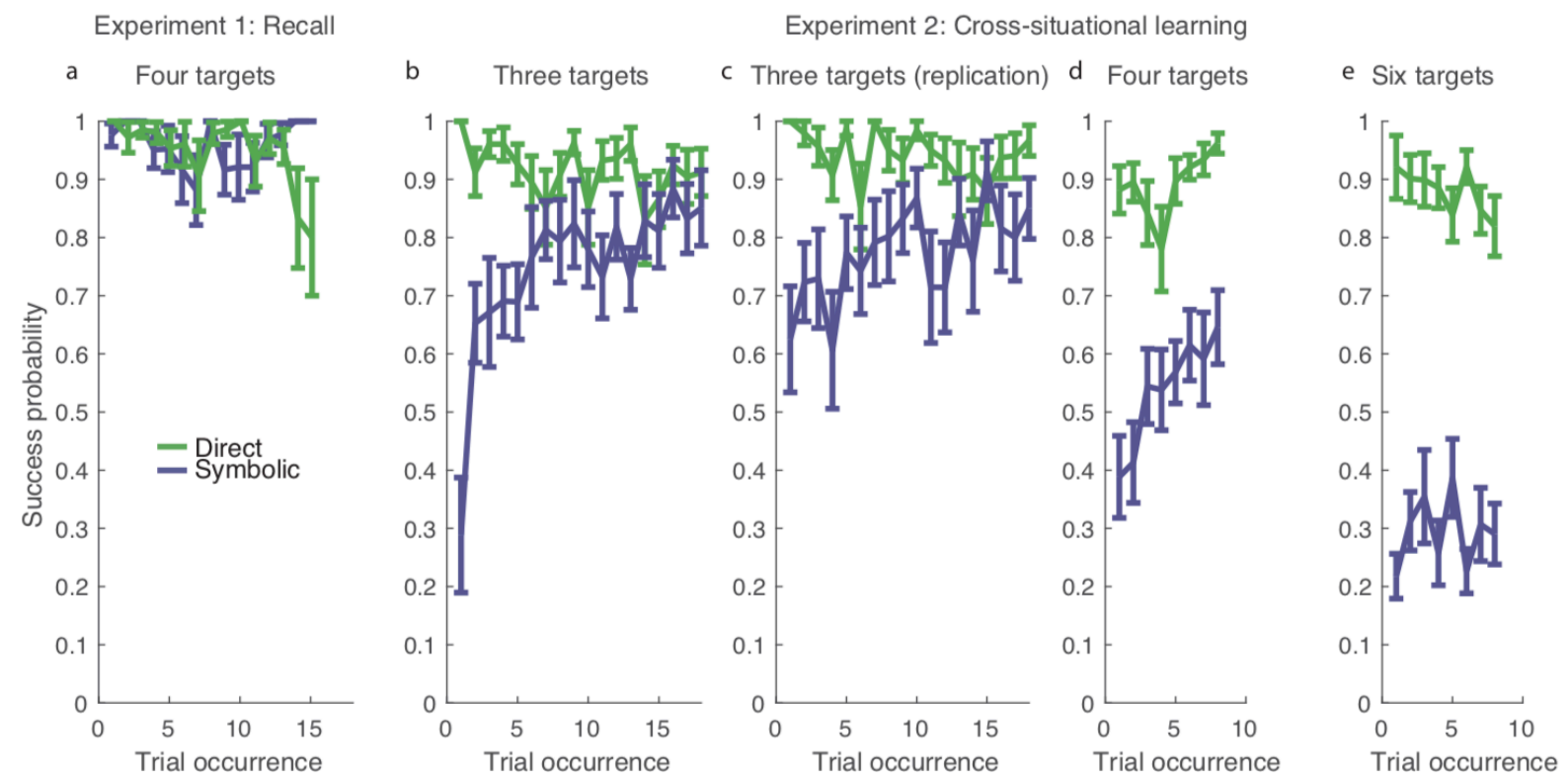

Figure 6: The success probability when the movement PT was below an individual's minimum PT, as a function of the cumulative number of exposures to a symbol in (a) Experiment 1, and in the (b) three-target, (c) three-target replication, (d) four-target, and (e) six-target variants of Experiment 2. For comparison, analogous curves for Direct pre-cued trials are shown as a function of target occurrences (in green).

$\mathrm{p}<0.0001 ; \alpha=-0.77, \mathrm{t}=-3.9, \mathrm{p}<0.001)$. In contrast, there was little evidence of learning in the six-target variant $(\beta=0.31, \mathrm{t}=13, \mathrm{p}<0.0001 ; \alpha=-1.46, \mathrm{t}=1.8, \mathrm{p}=0.079)$.

Explicit memory for the learned associations was tested through a symbol-target matching quiz. In all three variants of Experiment 2, the mean test accuracy closely matched the probability of making a correct reach when pre-cued with a symbol (Figure 5). In the replication study of the three-target variant (Figure 5b), which contained a memory test after the first block and at the end of the experiment, again the test accuracy closely matched reaching performance at the corresponding block.

Although we ran variants of the experiment with different numbers of targets/symbols to manipulate learning complexity, this permitted additional analyses. In particular, choice reaction 


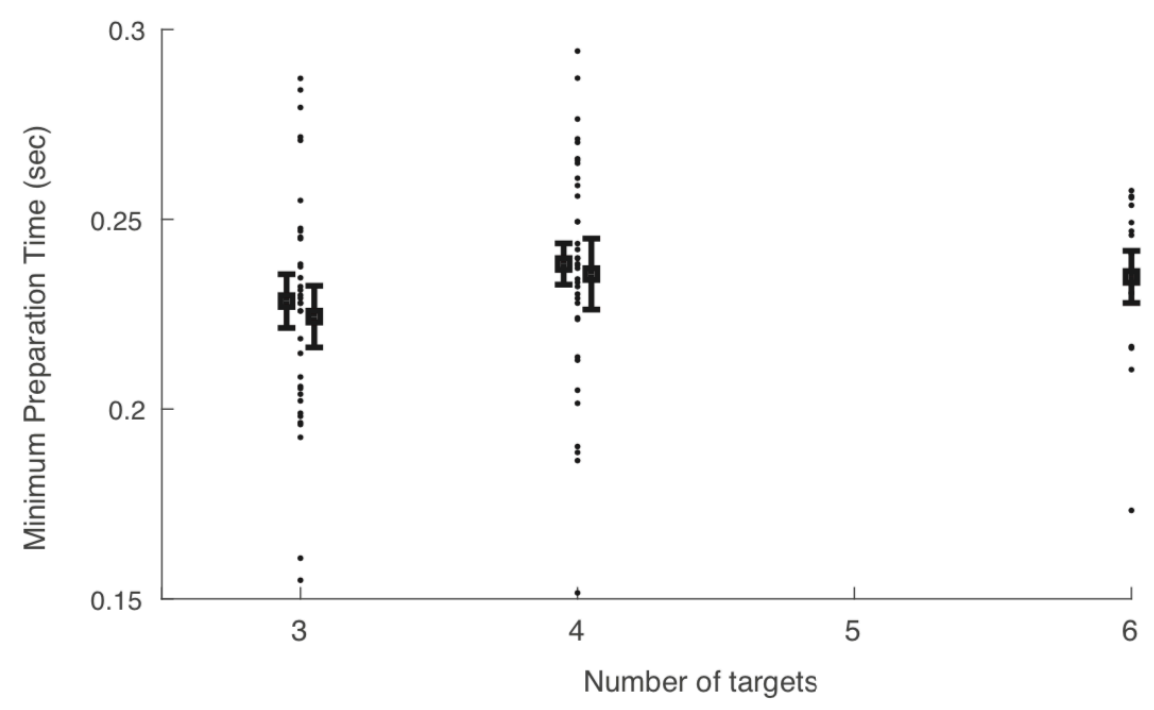

Figure 7: The minimum PT of reaches with no pre-cues as a function of the number of alternative potential targets. Black squares indicate the group average for an individual experiment or variant, and black dots indicate individual participants.

times are known to scale with the number of alternative choices according to a power law (Hick, 1952). Does Hick's Law apply to movement preparation when there are multiple potential targets? To answer this question, we conducted an analysis across all experiments of how the number of alternative targets affected the minimum PT required to successfully plan a movement when no pre-cue was given (Figure 7). A linear model fit to the minimum PT as a function of the number of targets failed to produce evidence of a relationship $(t=0.96, p=0.34)$. This null result is an apparent violation of Hick's law.

\section{Discussion}

An important component to learning novel motor behaviors is discovering and remembering the perceptual signs that predict upcoming movement requirements. We introduced a novel visuomotor association (VMA) task that enabled us to measure the movement response to visual cues while controlling for potential confounds inherent in other versions of the task. To avoid 
speed-accuracy tradeoffs, we controlled the RT on each trial using the timed response method. This allowed us to more definitively determine whether, and over what timecourse, visuomotor associations were learned and affected movement preparation.

Perceptual cues to movement

Experiment 1 tested whether recently memorized arbitrary visual cues would facilitate movement preparation to be as efficient as directly previewing the movement target. Movement goal uncertainty has been shown to delay reaction time (Scherbaum et al., 2010; Simon, 1969) and increase across-trial movement variability (Krüger \& Hermsdörfer, 2019; Song \& Nakayama, 2009). To quantify movement preparation, after controlling for RT, we measured success probability and movement variability. There were no detectable differences between direct precues versus symbolic pre-cues, and both conditions were superior compared to when no precues were available. The kinematics of the actual movements were otherwise similar across conditions. Any effect of preparation time on success probability or movement variability was small when either type of pre-cue was given. In contrast, when no pre-cue was given, an additional $200 \mathrm{~ms}$ of preparation time was needed to prepare reaches to the correct target.

Indeed, when no pre-cue was given and the preparation time was below an individual's minimum PT, this constituted a "go before you know" design (Chapman et al., 2010; Ghez et al., 1997; Haith et al., 2015). Trials with no pre-cue thus had a strong and significant effect of PT on success probability and movement variability, while trials with either direct or symbolic pre-cues had much weaker reliance on PT, providing further conclusive evidence that movements were planned in advance and equally well for both of the pre-cuing conditions. 
These findings confirmed our hypotheses that recently memorized associations between arbitrary symbols and targets would facilitate movement preparation equally as well as direct cues. This evidence highlights the importance of explicit memory for motor skill learning, as efficient movement preparation in advance of the appearance of movement goals is a critical component to many real-world skills (Bahill et al., 2005).

\section{Delays when misdirected}

An additional feature of Experiment 1 was that $10 \%$ of trials were catch trials, where a different target appeared than had been cued. Similar experimental designs, in which a change in movement plan is induced through a change in movement goals, have been used to study movement preparation (Ames et al., 2014, 2019; Haith et al., 2015; Selen et al., 2012). In our study, catch trials significantly delayed the minimum time that was necessary to successfully reach toward the presented target. This provides additional insight into the timecourse of movement preparation and further demonstrates that pre-cues resulted in movements being planned toward the cued target.

\section{Dynamics of learning}

Prior studies examined how actions are planned when prompted with an arbitrary visual cue (Asaad et al., 1998; Murray \& Wise, 1996; Wirth et al., 2003), but the mechanisms of early VMA learning have not specifically been characterized. We hypothesized that memorized associations would require no additional learning to be successfully deployed in a reaching task. An analysis of the timecourse of performance across trials in Experiment 1, where the VMAs were instructed and memorized ahead of time, revealed that there were no changes across practice. This demonstrates that the translation of known perceptual cues into movement goals does not 
require practice. Indeed, this facilitation seemed entirely mnemonic in nature, as target-choice behavior closely matched explicit recall accuracy in a post-test. These results give credence to the idea that motor learning is a cognitive activity (Stanley \& Krakauer, 2013).

We also hypothesized that VMAs can be learned de novo in a reaching task via a process akin to cross-situational learning (Aslin \& Newport, 2012; Frank et al., 2007; Nissen \& Bullemer, 1987; Yu \& Smith, 2007), from the repeated pairings of particular perceptual cues and movement goals. In Experiment 2, we ran three variants of such a cross-situational learning design. Participants exhibited a learning curve that was well-fit by an exponential function. These dynamics in learning the symbol-target associations led to a gradual improvement in the success probability across practice. The number of symbols associated with each target, the number of potential targets, and the symbol complexity each modulated the rate of learning.

Possible neural underpinnings

What mechanisms account for learning visuomotor associations over time? We found exponential dynamics of learning, consistent with a previous VMA study in non-human primates (Brasted et al., 2003). In that study, transection of the fornix, and thus deactivation of hippocampal contributions to recall, impaired VMA learning, suggesting a role for the hippocampus (see also Murray and Wise, 1996). Indeed, both the acquisition and expression of associations from cross-situational learning and statistical learning have been linked to the hippocampus (Berens et al., 2018; Covington et al., 2018; Eichenbaum, 2000; Schapiro et al., 2014; Wirth et al., 2003). This suggests that the hippocampus may be important for linking and recalling a movement goal for an arbitrarily perceptual cue. 
Although convention holds that motor learning is independent of the hippocampus (Corkin, 1968; Milner, 1962), recent research has suggested otherwise (Roy \& Park, 2010; Stanley \& Krakauer, 2013). Hippocampal-dependent processes might be necessary in motor learning through its capacity to memorize and learn associations, and to facilitate cortical processing (Kok et al., 2013; Kok \& Turk-Browne, 2018). This model of hippocampally mediated movement planning could underlie many motor behavior phenomena, such as recent evidence that memory recall enables faster adaptation to visuomotor perturbations (Huberdeau, Haith, et al., 2015) and contributes to motor sequence learning (Wong et al., 2015). A potentially fruitful direction for future research using this paradigm and conceptual model could be to examine brain activity changes in the hippocampus and other brain areas such as motor cortex across the learning and use of visuomotor associations.

\section{Violations of Hick's Law}

An intriguing, and unexpected, finding from this experiment was that the minimum PT when no pre-cue was given did not vary by the number of alternative target choices in Experiment 2. This finding violates expectations based on Hick's law (Hick, 1952) - the observation that reaction time scales as a function of the number of alternative choices (Christina et al., 1982; Fischman, 1984; Henry \& Rogers, 1960). An important methodological difference between the experiments presented here and previous findings is the use of the timed-response method, which forced responses across varying response times (i.e. the time between the presentation of a target and the actual movement onset). We expect that had the experiment allowed RT to vary freely, RT would have scaled with the number of potential targets when no pre-cue was given. This finding suggests that the origins of Hick's Law resides in deliberative processes, not motor preparation. 


\section{Limitations and future directions}

The VMA task that we used involved only ballistic arm reaches, whereas many human motor skills, including the opening example of baseball batters, involve continuous and complex movements. If movement is ongoing, how does visual information update the unfolding action? Some authors have addressed this question with tasks in which a stream of perceptual information is updated continuously (Selen et al., 2012). However, the learning and use of arbitrary perceptual cues has not been studied for continuous movements.

Our results raise the question of what exactly is the point of practice for motor skills. We showed that having knowledge of the associations resulted in an immediate benefit to performance in terms of the accuracy of action selection. The learning that was evident in the variants of Experiment 2 was still consistent with a mnemonic process: the probability of selecting the correct response was no different when tested with an explicit memory test versus with the VMA task. What then is the benefit of practice? First, the predicate circumstances that predict movement goals in many natural tasks might be far more complex to decipher than the simple and isolated stimuli used in the experiments presented here. Indeed, we found that increasing the visual complexity of symbols and the number of paired associations modulated the learning rate considerably. Had more practice been given in the four-and six-target variants of Experiment 2, additional learning might have occurred to bring asymptotic performance closer to the level observed for the three-target variant. Thus, significant practice may be needed by memory and cognitive systems to fully learn complex associations between perceptual cues and movement goals. Additionally, many real-world skills require more complex motor behaviors that might depend on a qualitatively different learning mechanism (Shmuelof et al., 2014). 


\section{References}

Ames, K. C., Ryu, S. I., \& Shenoy, K. V. (2014). Neural dynamics of reaching following incorrect or absent motor preparation. Neuron, 81(2), 438-451.

https://doi.org/10.1016/j.neuron.2013.11.003

Ames, K. C., Ryu, S. I., \& Shenoy, K. V. (2019). Simultaneous motor preparation and execution in a last-moment reach correction task. Nature Communications, 10(1), 2718. https://doi.org/10.1038/s41467-019-10772-2

Asaad, W. F., Rainer, G., \& Miller, E. K. (1998). Neural Activity in the Primate Prefrontal Cortex during Associative Learning. Neuron, 21(6), 1399-1407. https://doi.org/10.1016/S0896$6273(00) 80658-3$

Aslin, R. N., \& Newport, E. L. (2012). Statistical Learning: From Acquiring Specific Items to Forming General Rules. Current Directions in Psychological Science, 21(3), 170-176. https://doi.org/10.1177/0963721412436806

Bahill, A. T., Baldwin, D. G., \& Venkateswaran, J. (2005). Predicting a Baseball's Path: A batter watches the pitcher's motion plus the spin on the ball to calculate when and where it will cross the plate. American Scientist, 93(3), 218-225. https://doi.org/10.2307/27858576

Bédard, P., \& Sanes, J. N. (2009). On a basal ganglia role in learning and rehearsing visual-motor associations. Neurolmage, 47(4), 1701-1710. https://doi.org/10.1016/j.neuroimage.2009.03.050 
Berens, S. C., Horst, J. S., \& Bird, C. M. (2018). Cross-Situational Learning Is Supported by Propose-but-Verify Hypothesis Testing. Current Biology, 28(7), 1132-1136.e5. https://doi.org/10.1016/j.cub.2018.02.042

Brasted, P. J., Bussey, T. J., Murray, E. A., \& Wise, S. P. (2003). Role of the hippocampal system in associative learning beyond the spatial domain. Brain, 126(5), 1202-1223. https://doi.org/10.1093/brain/awg103

Chapman, C. S., Gallivan, J. P., Wood, D. K., Milne, J. L., Culham, J. C., \& Goodale, M. A. (2010). Reaching for the unknown: Multiple target encoding and real-time decision-making in a rapid reach task. Cognition, 116(2), 168-176.

https://doi.org/10.1016/j.cognition.2010.04.008

Christina, R. W., Fischman, M. G., Vercruyssen, M. J. P., \& Anson, J. G. (1982). Simple Reaction Time as a Function of Response Complexity. Journal of Motor Behavior, 14(4), 301-321. https://doi.org/10.1080/00222895.1982.10735282

Collins, A. G. E., \& Frank, M. J. (2012). How much of reinforcement learning is working memory, not reinforcement learning? A behavioral, computational, and neurogenetic analysis. European Journal of Neuroscience, 35(7), 1024-1035. https://doi.org/10.1111/j.14609568.2011.07980.x

Corkin, S. (1968). Acquisition of motor skill after bilateral medial temporal-lobe excision. Neuropsychologia, 6(3), 255-265. https://doi.org/10.1016/0028-3932(68)90024-9

Covington, N. V., Brown-Schmidt, S., \& Duff, M. C. (2018). The Necessity of the Hippocampus for Statistical Learning. Journal of Cognitive Neuroscience, 30(5), 680-697. https://doi.org/10.1162/jocn_a_01228 
Dang, J. S., Figueroa, I. J., \& Helton, W. S. (2018). You are measuring the decision to be fast, not inattention: The Sustained Attention to Response Task does not measure sustained attention. Experimental Brain Research, 236(8), 2255-2262. https://doi.org/10.1007/s00221-018-5291-6

Doll, B. B., Shohamy, D., \& Daw, N. D. (2015). Multiple memory systems as substrates for multiple decision systems. Neurobiology of Learning and Memory, 117, 4-13. https://doi.org/10.1016/j.nlm.2014.04.014

Eichenbaum, H. (2000). A cortical-hippocampal system for declarative memory. Nature Reviews Neuroscience, 1(1), 41-50. https://doi.org/10.1038/35036213

Fischman, M. G. (1984). Programming Time as a Function of Number of Movement Parts and Changes in Movement Direction. Journal of Motor Behavior, 16(4), 405-423. https://doi.org/10.1080/00222895.1984.10735329

Frank, M., Goodman, N. D., \& Tenenbaum, J. B. (2007). A Bayesian Framework for CrossSituational Word-Learning. Neural Information Processing Systems.

Ghez, C., Favilla, M., Ghilardi, M. F., Gordon, J., Bermejo, R., \& Pullman, S. (1997). Discrete and continuous planning of hand movements and isometric force trajectories. Experimental Brain Research, 115(2), 217-233. https://doi.org/10.1007/PL00005692

Haith, A. M., Huberdeau, D. M., \& Krakauer, J. W. (2015). Hedging Your Bets: Intermediate Movements as Optimal Behavior in the Context of an Incomplete Decision. PLoS Comput Biol, 11(3), e1004171. https://doi.org/10.1371/journal.pcbi.1004171 
Haith, A. M., Pakpoor, J., \& Krakauer, J. W. (2016). Independence of Movement Preparation and Movement Initiation. The Journal of Neuroscience: The Official Journal of the Society for Neuroscience, 36(10), 3007-3015. https://doi.org/10.1523/JNEUROSCI.3245-15.2016

Hardwick, R. M., Forrence, A. D., Krakauer, J. W., \& Haith, A. M. (2017). Skill Acquisition and Habit Formation as Distinct Effects of Practice. BioRxiv, 201095. https://doi.org/10.1101/201095

Henry, F. M., \& Rogers, D. E. (1960). Increased Response Latency for Complicated Movements and A "Memory Drum" Theory of Neuromotor Reaction. Research Quarterly. American Association for Health, Physical Education and Recreation, 31(3), 448-458. https://doi.org/10.1080/10671188.1960.10762052

Hick, W. E. (1952). On the rate of gain of information. Quarterly Journal of Experimental Psychology, 4(1), 11-26.

Huberdeau, D. M., Haith, A. M., \& Krakauer, J. W. (2015). Formation of a long-term memory for visuomotor adaptation following only a few trials of practice. Journal of Neurophysiology, 114(2), 969-977. https://doi.org/10.1152/jn.00369.2015

Huberdeau, D. M., Krakauer, J. W., \& Haith, A. M. (2015). Dual-process decomposition in human sensorimotor adaptation. Current Opinion in Neurobiology, 33, 71-77. https://doi.org/10.1016/j.conb.2015.03.003

Hunt, R. H., \& Aslin, R. N. (2001). Statistical learning in a serial reaction time task: Access to separable statistical cues by individual learners. Journal of Experimental Psychology. General, 130(4), 658-680. 
Knowlton, B. J., Mangels, J. A., \& Squire, L. R. (1996). A Neostriatal Habit Learning System in Humans. Science, 273(5280), 1399-1402. https://doi.org/10.1126/science.273.5280.1399

Kok, P., Brouwer, G. J., Gerven, M. A. J. van, \& Lange, F. P. de. (2013). Prior Expectations Bias Sensory Representations in Visual Cortex. Journal of Neuroscience, 33(41), 1627516284. https://doi.org/10.1523/JNEUROSCI.0742-13.2013

Kok, P., \& Turk-Browne, N. B. (2018). Associative Prediction of Visual Shape in the Hippocampus. Journal of Neuroscience, 38(31), 6888-6899. https://doi.org/10.1523/JNEUROSCI.0163-18.2018

Krüger, M., \& Hermsdörfer, J. (2019). Target Uncertainty During Motor Decision-Making: The Time Course of Movement Variability Reveals the Effect of Different Sources of Uncertainty on the Control of Reaching Movements. Frontiers in Psychology, 10. https://doi.org/10.3389/fpsyg.2019.00041

Liu, C. C., \& Watanabe, T. (2012). Accounting for speed-accuracy tradeoff in perceptual learning. Vision Research, 61, 107-114. https://doi.org/10.1016/j.visres.2011.09.007 McDougle, S. D., \& Taylor, J. A. (2019). Dissociable cognitive strategies for sensorimotor learning. Nature Communications, 10(1), 40. https://doi.org/10.1038/s41467-01807941-0

McElree, B., Murphy, G. L., \& Ochoa, T. (2006). Time-Course of Retrieving Conceptual Information: A Speed-Accuracy Tradeoff Study. Psychonomic Bulletin \& Review, 13(5), 848-853. 
Milner, B. (1962). Les troubles de la memoire accompagnant des lesions hippocampiques bilaterales. In Physiologie de l'hippocampe (pp. 257-272). Centre National de la Recherche Scientifique.

Murray, E. A., \& Wise, S. P. (1996). Role of the Hippocampus Plus Subjacent Cortex but Not Amygdala in Visuomotor Conditional Learning in Rhesus Monkeys. Behavioral Neuroscience, 110(6), 1261-1270. https://doi.org/10.1037/0735-7044.110.6.1261

Nissen, M. J., \& Bullemer, P. (1987). Attentional requirements of learning: Evidence from performance measures. Cognitive Psychology, 19(1), 1-32. https://doi.org/10.1016/0010-0285(87)90002-8

Peebles, D., \& Bothell, D. (2014). Modelling Performance in the Sustained Attention to Response Task. Proc ICCM, 231-236.

Pew, R. W. (1969). The speed-accuracy operating characteristic. Acta Psychologica, 30, 16-26. https://doi.org/10.1016/0001-6918(69)90035-3

Roy, S., \& Park, N. W. (2010). Dissociating the memory systems mediating complex tool knowledge and skills. Neuropsychologia, 48(10), 3026-3036. https://doi.org/10.1016/j.neuropsychologia.2010.06.012

Schapiro, A. C., Gregory, E., Landau, B., McCloskey, M., \& Turk-Browne, N. B. (2014). The Necessity of the Medial Temporal Lobe for Statistical Learning. Journal of Cognitive Neuroscience, 26(8), 1736-1747. https://doi.org/10.1162/jocn_a_00578

Scherbaum, S., Dshemuchadse, M., Fischer, R., \& Goschke, T. (2010). How decisions evolve: The temporal dynamics of action selection. Cognition, 115(3), 407-416. https://doi.org/10.1016/j.cognition.2010.02.004 
Selen, L. P. J., Shadlen, M. N., \& Wolpert, D. M. (2012). Deliberation in the Motor System: Reflex Gains Track Evolving Evidence Leading to a Decision. The Journal of Neuroscience, 32(7), 2276-2286. https://doi.org/10.1523/JNEUROSCI.5273-11.2012

Shmuelof, L., Yang, J., Caffo, B., Mazzoni, P., \& Krakauer, J. W. (2014). The neural correlates of learned motor acuity. Journal of Neurophysiology, 112(4), 971-980. https://doi.org/10.1152/jn.00897.2013

Simon, J. R. (1969). Reactions toward the source of stimulation. Journal of Experimental Psychology, 81(1), 174-176.

Smith, L. B., \& Yu, C. (2013). Visual attention is not enough: Individual differences in statistical word-referent learning in infants. Language Learning and Development: The Official Journal of the Society for Language Development, 9(1). https://doi.org/10.1080/15475441.2012.707104

Song, J.-H., \& Nakayama, K. (2009). Hidden cognitive states revealed in choice reaching tasks. Trends in Cognitive Sciences, 13(8), 360-366. https://doi.org/10.1016/j.tics.2009.04.009

Squire, L. R. (1992). Declarative and nondeclarative memory: Multiple brain systems supporting learning and memory. Journal of Cognitive Neuroscience, 4(3), 232-243. https://doi.org/10.1162/jocn.1992.4.3.232

Squire, Larry R., \& Zola, S. M. (1996). Structure and function of declarative and nondeclarative memory systems. Proceedings of the National Academy of Sciences, 93(24), 1351513522.

Stanley, J., \& Krakauer, J. W. (2013). Motor skill depends on knowledge of facts. Frontiers in Human Neuroscience, 7. https://doi.org/10.3389/fnhum.2013.00503 
Sutton, R. S., \& Barto, A. G. (1998). Introduction to reinforcement learning. MIT Press.

Trapp, S., Lepsien, J., Sehm, B., Villringer, A., \& Ragert, P. (2012). Changes of hand switching costs during bimanual sequential learning. PloS One, 7(9), e45857. https://doi.org/10.1371/journal.pone.0045857

Wirth, S., Yanike, M., Frank, L. M., Smith, A. C., Brown, E. N., \& Suzuki, W. A. (2003). Single Neurons in the Monkey Hippocampus and Learning of New Associations. Science, 300(5625), 1578-1581. https://doi.org/10.1126/science.1084324

Wong, A. L., Goldsmith, J., Forrence, A. D., Haith, A. M., \& Krakauer, J. W. (2017). Reaction times can reflect habits rather than computations. ELife, 6, e28075. https://doi.org/10.7554/eLife.28075

Wong, A. L., Lindquist, M. A., Haith, A. M., \& Krakauer, J. W. (2015). Explicit knowledge enhances motor vigor and performance: Motivation versus practice in sequence tasks. Journal of Neurophysiology, 114(1), 219-232. https://doi.org/10.1152/jn.00218.2015

Wood, C. C., \& Jennings, J. R. (1976). Speed-accuracy tradeoff functions in choice reaction time: Experimental designs and computational procedures. Perception \& Psychophysics, 19(1), 92-102. https://doi.org/10.3758/BF03199392

Yu, C., \& Smith, L. (2011). What You Learn is What You See: Using Eye Movements to Study Infant Cross-Situational Word Learning. Developmental Science, 14(2), 165-180.

Yu, C., \& Smith, L. B. (2007). Rapid Word Learning Under Uncertainty via Cross-Situational Statistics. Psychological Science, 18(5), 414-420. https://doi.org/10.1111/j.14679280.2007.01915.x 


\section{Supplemental Figure 1}

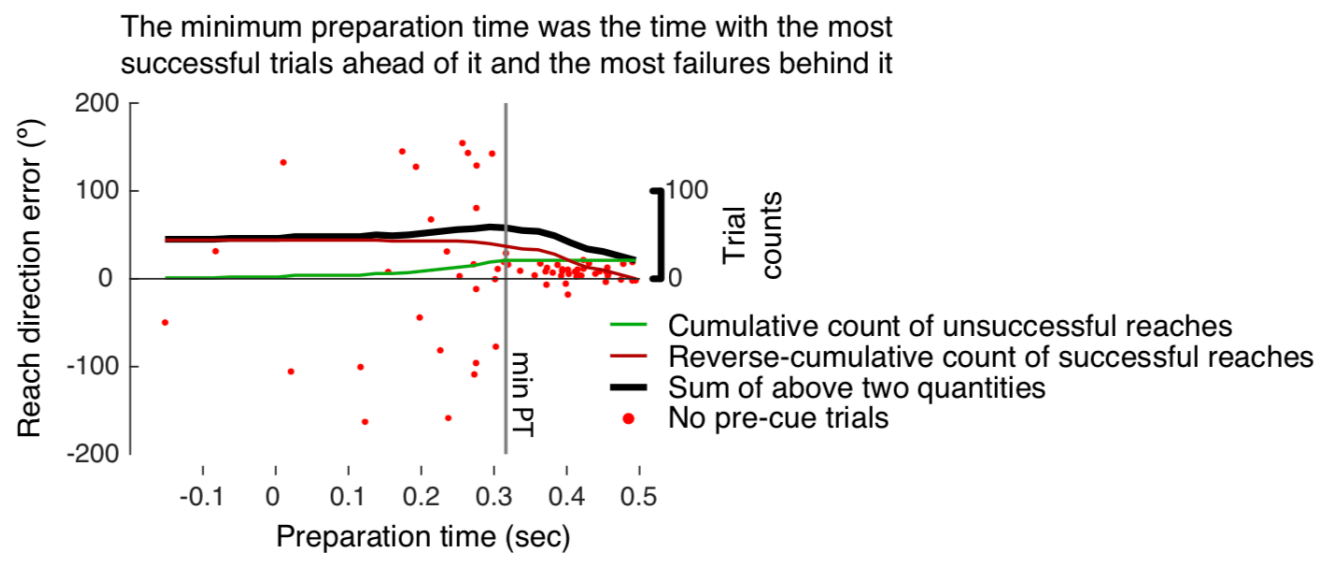

Supplemental Figure 1: Calculating an individual's minimum PT. The cumulative sum of non-correct trials and the reverse-cumulative sum of correct trials were summed to get a function that peaked near the minimum PT. Given the lack of resolution of trials precisely at the minimum PT for many participants, the minimum PT was taken as the latest time at which this function was within $95 \%$ of its maximum. 